\title{
Matrix Cosmology
}

\section{Citation}

Karczmarek, Joanna L, and Andrew Strominger. 2004. "Matrix Cosmology." Journal of High Energy Physics 2004 (4): 055-055. https://doi.org/10.1088/1126-6708/2004/04/055.

\section{Permanent link}

http://nrs.harvard.edu/urn-3:HUL.InstRepos:41417354

\section{Terms of Use}

This article was downloaded from Harvard University's DASH repository, and is made available under the terms and conditions applicable to Other Posted Material, as set forth at http:// nrs.harvard.edu/urn-3:HUL.InstRepos:dash.current.terms-of-use\#LAA

\section{Share Your Story}

The Harvard community has made this article openly available.

Please share how this access benefits you. Submit a story.

Accessibility 
hep-th/0309138

\title{
Matrix Cosmology
}

\author{
Joanna L. Karczmarek and Andrew Strominger \\ Jefferson Physical Laboratory, Harvard University, Cambridge, MA 02138
}

\begin{abstract}
Exact time-dependent solutions of $c=1$ string theory are described using the free fermion formulation. One such class of solutions describes draining of the Fermi sea and has a spacetime interpretation as closed string tachyon condensation. A second class of solutions, corresponding to droplets of Fermi liquid orbiting in phase space, describes closed cosmologies which bounce through singularities.
\end{abstract}




\section{Contents}

1. Introduction . . . . . . . . . . . . . . . . . . . . . . . . . . . . 1

2. Lightning review of the $c=1$ matrix model . . . . . . . . . . . . . . . . . . . . 2

3. Tachyon condensation and draining the Fermi sea . . . . . . . . . . . . . . . . . 4

3.1. Lightlike condensation . . . . . . . . . . . . . . . . . . . . . . . . . . . 5

3.2. Spacelike condensation . . . . . . . . . . . . . . . . . . . . . . . . . . . 8

3.3. Exact quantum solutions . . . . . . . . . . . . . . . . . . . . . . . . . . 10

3.4. Stability . . . . . . . . . . . . . . . . . . . . . . . . . . . . . . . . . 11

4. Closed cosmologies . . . . . . . . . . . . . . . . . . . . . . . . . . . . . . . 11

4.1. Orbiting Fermi droplet . . . . . . . . . . . . . . . . . . . . . . . . . . . 11

4.2. Validity of the semiclassical approximation . . . . . . . . . . . . . . . . . . 15

5. General droplet cosmology . . . . . . . . . . . . . . . . . . . . . . . . . . . 16

6. SD-branes and higher dimensions . . . . . . . . . . . . . . . . . . . . . . . . 18

\section{Introduction}

The discovery of the double scaling limit of $c \leq 1$ matrix models [1-6] provided a beautiful and nonperturbative solution to bosonic string theory in two spacetime dimensions [7]. However these developments seemed not to fully find their place within the modern framework of string duality. Recently, the situation has changed with the elegant reinterpretation of these models as a soluble example of bulk-boundary duality [8 10] for type 0 strings [11, 12]. With this new perspective it is natural to try to use these models to address various outstanding puzzles in nonperturbative string theory. In this paper we will look at time-dependent solutions of $c=1$ string theory.

The mathematically simplest formulation of the $c=1$ theory is as a theory of free fermions in an inverted harmonic oscillator potential. In this setup the ground state is usually described as having the Fermi sea filled up to energy $-\mu$, where $\mu$ is related to the string coupling. The point of view which we shall adopt in this paper is that the full nonperturbative theory contains all states of any number of free fermions in the inverted harmonic potential. These states may or may not have a weakly coupled description as a smooth $D=2$ spacetime geometry with tachyons. However, we find several examples in which such weakly coupled descriptions can be derived for arbitrarily large regions of spacetime, while other regions contain singularities which are resolved in the free fermion description. Our analysis relies heavily on the tachyon-fermion dictionary given in [13, 14]. 
One class of examples involves tachyon condensation along a spacelike 1 or null hypersurface. $⿴$ The spacelike case at weak coupling corresponds to a worldsheet with a timelike bulk Liouville factor, while the null case involves (an analytic continuation of) the SineGordon Liouville theory. Both have exact descriptions in the free fermion picture as a draining of the Fermi sea. A second class of solutions describes closed, ekpyrotic cosmologies which begin at weak coupling and bounce off a strong coupling region into a second weakly-coupled region. This is described as a finite-size but macroscopic droplet of the Fermi sea orbiting in phase space.

This paper is organized as follows. Section 2 briefly reviews the $c=1$ matrix model and fixes our notation. Section 3 describes two classes of semiclassical solutions of the free Fermi theory, characterized as a moving liquid in phase space. It is then shown, using the dictionary of [13], that these correspond to tachyon condensation along null or spacelike slices. We further go beyond the semiclassical limit and construct exact quantum states in the free fermion picture corresponding to tachyon condensation. Section 4 contains a closed cosmology with a large weakly coupled region. Section 5 considers a more general class of cosmologies which correspond to arbitrarily shaped droplets of Fermi liquid orbiting in phase space. This project arose as a search for a large $N$ decoupling limit of sD-branes, and in the last section we describe how the Fermi droplets are a $c=1$ version of such, pointing out also potential generalizations to higher dimensions.

After initial submission of this paper, we became aware of work by S. Alexandrov, V. Kazakov and I. Kostov [16], which overlaps with our paper. See also [17-19] for related interesting results.

\section{Lightning review of the $c=1$ matrix model}

In the free fermion formulation the $c=1$ matrix model is described by the hamiltonian 3

$$
\mathcal{H}=\frac{1}{2} \int d x\left(\partial_{x} \Psi^{\dagger} \partial_{x} \Psi-x^{2} \Psi^{\dagger} \Psi\right)
$$

where

$$
\left\{\Psi^{\dagger}(x), \Psi\left(x^{\prime}\right)\right\}=\delta\left(x-x^{\prime}\right)
$$

1 The $g_{s} \rightarrow \infty$ limit of our solution first appeared in [15].

2 This involves a non-normalizable perturbation, and hence does not ruin the stability of the usual ground state.

3 We set $\alpha^{\prime}=1$ and roughly follow the notation of [20]. 
The usual ground state $|\mu\rangle$ is defined by filling the Fermi sea up to an energy $E=-\mu$ :

$$
b_{E}^{\dagger}|\mu\rangle=0, \quad E<-\mu, \quad b_{E}|\mu\rangle=0, \quad E>-\mu,
$$

where $b_{E}^{\dagger}$ creates a fermion of energy $E$. This requires an infinite number of fermions since the energy in the inverted harmonic oscillator is unbounded from below.

For part of our discussion we will be expanding about the classical point particle limit in which the phase space volume per fermion goes to zero and the Fermi sea can be treated as a continuous liquid in phase space [13]. The Fermi sea and its boundary the Fermi surface move along classical trajectories - hyperbolas in phase space - under time evolution. The ground state corresponds to filling the phase space region bounded by the classical trajectory with $E=-\mu$ as depicted in figure 1(a). Since this boundary is invariant under time evolution, the ground state is static. We will be interested in more general time dependent states which may be semiclassically described by filling a region of phase space at $t=0$ and then evolving it along the classical trajectories.

The description of this system as a $c=1$ noncritical string theory contains a tachyon field $T$ propagating in two spacetime dimensions. This field is a bosonization of $\Psi$ describing fluctuations in the Fermi surface of the ground state $|\mu\rangle$. At any moment of time the value of the momentum $p$ on the Fermi surface is a double-valued function of $x$ whose values we denote $p_{ \pm}(x)$. In the ground state,

$$
p_{ \pm}= \pm \sqrt{x^{2}-g_{s}^{-1}}
$$

where the distance of closest approach to the origin is the inverse square root of the string coupling, $g_{s}=1 / 2 \mu$. More generally, the classical equations of motion

$$
\dot{p}=x, \quad \dot{x}=p
$$

imply

$$
\partial_{t} p_{ \pm}=x-\left(\partial_{x} p_{ \pm}\right) p_{ \pm}
$$

Following [13], $p_{ \pm}$may be written in terms of a collective field $S$ and its canonical conjugate $\pi_{S}:$

$$
p_{ \pm}= \pm e^{-q}-\sqrt{\pi} e^{q}\left(\pi_{S} \mp \partial_{q} S\right),
$$

where $x=-e^{-q}$. To match (2.4), $S$ at large negative $q$ must be

$$
S(q)=-\frac{q}{2 \sqrt{\pi} g_{s}}+\text { const }
$$


The collective field $S$ is related to the tachyon $T$ in the Liouville theory by $⿴$

$$
T \propto e^{2 q} S
$$

This correspondence is meaningful only for $x \rightarrow-\infty$, where the worldsheet theory is weakly coupled. With $\alpha^{\prime}=1$, the coordinates $(q, t)$ are related to the Liouville coordinates $\left(X^{1}, X^{0}\right)$ by $X^{0}=t$ and $X^{1}=q+$ const. Following $[13$ 目 we choose the fudge factor in (2.9) and (2.8) so that the tachyon field corresponding to (2.8) is

$$
T=-\left(\hat{\mu} X^{1}\right) e^{2 X^{1}} .
$$

Including this field leads to the closed string worldsheet action

$$
\frac{1}{4 \pi} \int d^{2} \sigma \sqrt{\hat{g}}\left\{\hat{g}^{a b} \eta_{\mu \nu} \partial_{a} X^{\mu} \partial_{b} X^{\nu}+2 X^{1} \hat{R}-\hat{\mu} X^{1} e^{2 X^{1}}\right\}
$$

which is the $c=25$ Liouville theory supplemented by a timelike boson.

A small incoming tachyon fluctuation can be described as a bump in the Fermi sea which makes its way around the hyperbola. Using this picture and the relation (2.7) the tachyon S-matrix has been computed from the free fermion picture and compared to results from standard worldsheet methods based on (2.11). Agreement has been found - a review appears in [22,23].

\section{Tachyon condensation and draining the Fermi sea}

In this section, we will describe a simple time-dependent solution of the free fermion model (2.1). Using the dictionary (2.7), we will see that the deviation of the Fermi sea from the ground state (2.4) is too large for the solution to be interpreted as weak-field tachyon scattering. Instead we will argue that it corresponds to closed string tachyon condensation.

4 In work subsequent to [13], a distinction is made betweed the string theory field $S$ and the matrix model collective field $\bar{S}$ [21]. For our purposes, this distinction is not important, and we will not stress it. $\bar{S}$ and $S$ are related by a nonlocal transform which can be represented as a function of $d / d q$ acting on $\bar{S}$. For the specific forms of $S(q)$ which we will consider, this will reduce to either a constant multiplicative factor, or an additive constant.

5 Except for the constant shift allowed between $X^{1}$ and $q$ which has been chosen to simplify the expression. 


\subsection{Lightlike condensation}

A solution of the equations of motion for the Fermi surface is given by 6

$$
\left(x+p+2 \lambda_{+} e^{t}\right)\left(x-p+2 \lambda_{-} e^{-t}\right)=g_{s}^{-1},
$$

where $\lambda_{+}, \lambda_{-}$are arbitrary non-negative constants. This is a moving hyperbola centered at $(x, p)=\left(-\lambda_{+} e^{t}-\lambda_{-} e^{-t},-\lambda_{+} e^{t}+\lambda_{-} e^{-t}\right)$ rather than the origin.

To understand what (3.1) represents, let's consider some special cases. Take $\lambda_{+}=$ $\lambda_{-}=\lambda$; at $t=0$ the filled portion of the Fermi sea is displaced by an amount $-2 \lambda$ in the $x$ direction (see figure $1(\mathrm{c})$ ). Under time evolution fermions come in from $x=-\infty$, up to the point given by $x=-2 \lambda-1 / \sqrt{g_{s}}$ and then back to $x=-\infty$. Another interesting special case is $\lambda_{-}=0$. In the infinite past the Fermi sea is essentially static and filled up to the energy $g_{s}^{-1}$ below the top of the potential. It then decays away (rolls down the potential) and all fermions move out to $x=-\infty$ in the infinite future.

Using the dictionary (2.7)in the asymptotic region of large $-x=e^{-q}$, the solution (3.1) corresponds to

$$
\sqrt{\pi} S=-\frac{1}{2 g_{s}} q+\lambda_{+} e^{t-q}+\lambda_{-} e^{-t-q}+o\left(e^{-2 q}\right),
$$

up to a constant. According to (2.9), the tachyon field corresponding to (3.2) is $\mathrm{G}$

$$
T=-\hat{\mu} X^{1} e^{2 X^{1}}+\hat{\lambda}_{+} e^{X^{1}+X^{0}}+\hat{\lambda}_{-} e^{X^{1}-X^{0}},
$$

where $\hat{\lambda}_{ \pm}$are proportional to $\lambda_{ \pm}$. Including this field leads to the closed string worldsheet action

$$
\frac{1}{4 \pi} \int d^{2} \sigma \sqrt{\hat{g}}\left\{2 X^{1} \hat{R}-\hat{\mu} X^{1} e^{2 X^{1}}+\hat{\lambda}_{+} e^{X^{1}+X^{0}}+\hat{\lambda}_{-} e^{X^{1}-X^{0}}\right\} .
$$

6 For a Type 0 NS solution we should add a second filled region at positive $x$. While the existence of a nonperturbative Type 0 interpretation is important for our discussion, the details are not, and we shall for the sake of brevity suppress all discussion of the second region.

7 We suppress the effect of the leg-pole factors, which can be accounted for by a nonlocal transform, as in equation (20) of [21]. Here, the effect of this transform is just a multiplicative factor, which can be absorbed by a rescaling of the couplings and a shift in $X^{1}$. However, the kernel $\mathrm{K}$ has a pole for the precise solution we are considering, resulting in a divergence which must be regularized and renormalized. One regulator we can use is to slightly change the interaction term, moving its euclidian momentum away from the pole in the kernel, while keeping the resulting perturbation marginal. 


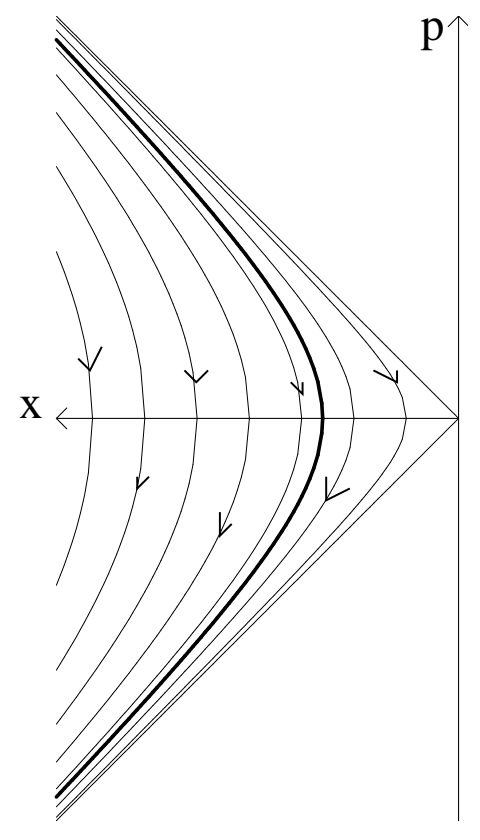

(a)

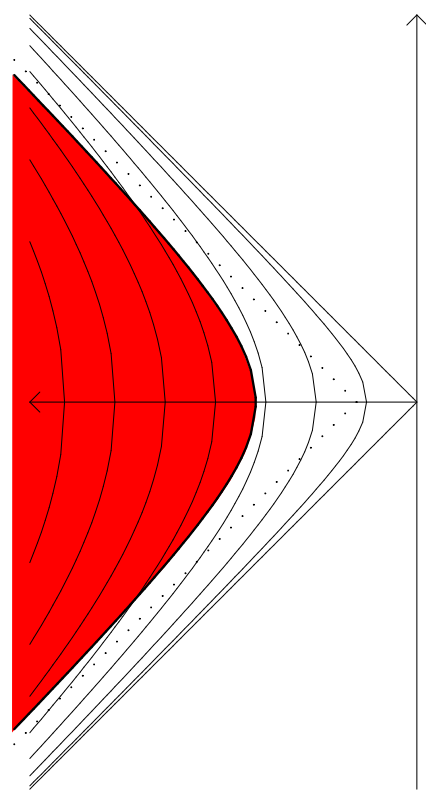

(c) $\mathrm{t}=0$

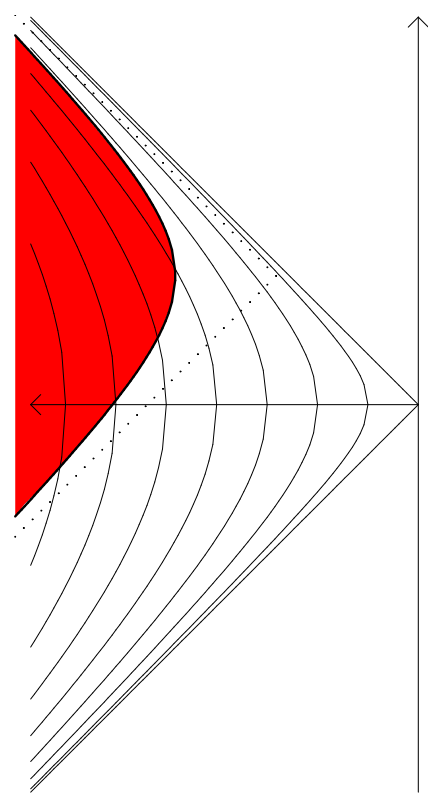

(b) $\mathrm{t}=-1.5$

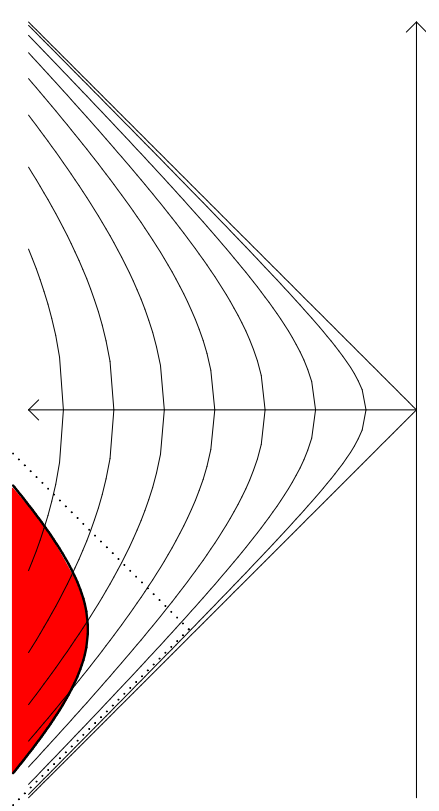

(d) $\mathrm{t}=2$

Fig. 1: (a) The usual ground state has the Fermi sea filled up to the left constant energy surface $E=-1 / g_{s}$ denoted by the bold hyperbola. The arrowed lines indicate classical trajectories. (b-d) At any fixed time the general solution (3.1) is a displaced hyperbola, each point of which which is dragged along the classical trajectories. 
Analytic continuation $X^{0} \rightarrow-i X^{2}$ of this action gives the Sine-Gordon Liouville action. The first interaction term corresponds to the standard Liouville potential which is a timelike "wall" at $X^{1} \sim-\ln \hat{\mu}$. The second and third interaction terms (which are weight $(1,1)$ operators) are also exponential potential walls, albeit less steep than the standard one. These walls move with time, effectively cutting off the universe at a time-varying distance. They are located roughly along the ingoing and outgoing null trajectories at $X^{1} \pm X^{0} \sim-\ln \hat{\lambda}_{ \pm}$. Hence at large positive (negative) $X^{0}$, the wall moves outwards (inwards) with essentially the speed of light. The situation is depicted in figure 2 .

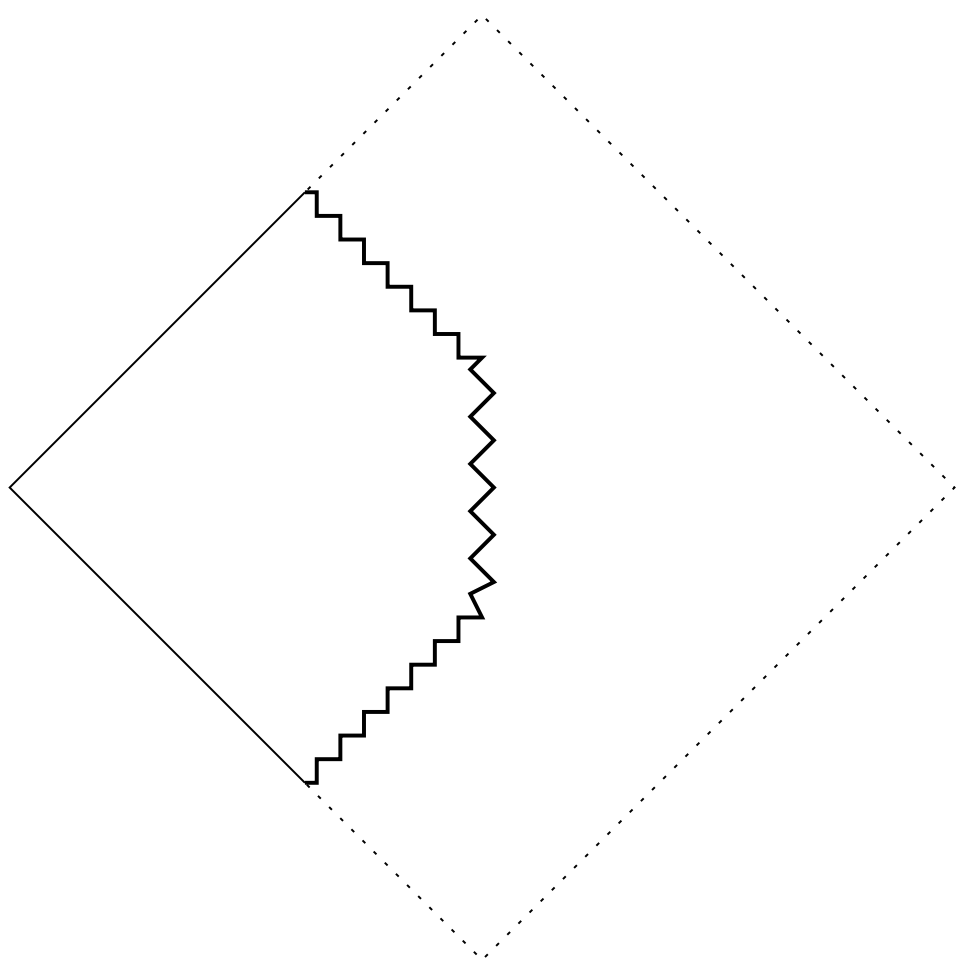

Fig. 2: Penrose diagram for lightlike tachyon condensation. The jagged lines indicate the locations of the past, future and timelike Liouville walls.

Any observer moving along a timelike trajectory will eventually move into a region where the tachyon field becomes arbitrarily large. Therefore this solution is a form of tachyon condensation. An exact expression for the quantum state corresponding to this solutions will be given in subsection 3.3.

A limit can be considered in which the first interaction term in (3.4) can be eliminated by setting $\hat{\mu}$ (and hence $g_{s}^{-1}$ ) to zero. This corresponds to a Fermi surface which is not a smooth moving hyperbola, but has a corner which itself moves along a hyperbola. The strong coupling region is now hidden by the null ingoing and outgoing potential walls. 
A further limit may be taken in which all but the last term are set to zero. In the far past, the remaining term vanishes and we have the linear dilaton vacuum with no Liouville wall. At any finite time a wall is present and moving out to spatial infinity at the speed of light. In the far future the tachyon has condensed and the fermions are all gone. This corresponds to draining of the Fermi sea. However, this limiting situation is singular in the tachyon description, since at $t=-\infty$ the strong coupling region is exposed.

\subsection{Spacelike condensation}

In this section, we present another method of draining the Fermi sea, which can also be interpreted as tachyon condensation, but on a spacelike surface.

The general solution is

$$
\left(\sinh \left(t-T_{1}\right) x-\cosh \left(t-T_{1}\right) p\right)\left(\sinh \left(t-T_{2}\right) x-\cosh \left(t-T_{2}\right) p\right)=-\frac{1}{2 g_{s}} \cosh \left(T_{2}-T_{1}\right) .
$$

The $g_{s} \rightarrow \infty$ limit of this solution appeared first in 150 . We first consider this solution in the $T_{1} \rightarrow-\infty, g_{s} \rightarrow \infty$ limit

$$
e^{t}(x-p)(\sinh (t) x-\cosh (t) p)=0 .
$$

This corresponds to a "wedge" of the Fermi sea. At $t=-\infty$, it is the entire quadrant $p+x>0, p-x<0$, which is the linear dilaton vacuum without a tachyon wall. As time passes, the upper edge of the Fermi sea remains a straight line, but rotates counterclockwise in such a fashion that it has slope $\tanh (t)$. At $t=+\infty$, the upper and lower edges coincide, and the Fermi sea has disappeared. This is depicted in figure 3. At early times the tachyon field is small and we can use (2.7) and (2.9) to compute its value. We find for $X^{0} \rightarrow-\infty$

$$
T \sim e^{2 X^{0}} .
$$

Unlike the previous case, here we find a tachyon field which is constant along spacelike (rather than null) slices. (3.7) is the exactly marginal timelike bulk Liouville interaction considered in [24-26].

The above case of pure linear dilaton is singular because there is no tachyon wall shielding the strong coupling region. This wall is reinstated by taking $g_{s} \neq 0$, so that

$$
e^{t}(x-p)(\sinh (t) x-\cosh (t) p)=-\frac{1}{2 g_{s}} .
$$

8 We thank J. Polchinski for drawing this to our attention. 


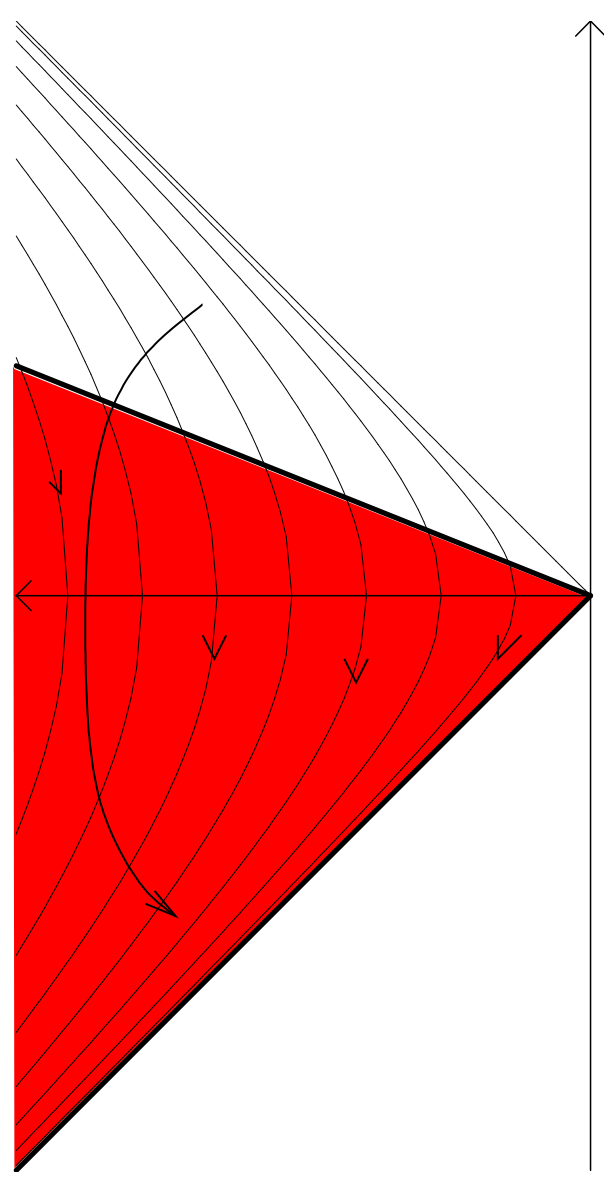

Fig. 3: Tachyon condensation on a spacelike surface in the linear dilaton vacuum is represented by the upper edge of the Fermi sea undergoing counterclockwise rotation. In the infinite future it meets the lower edge and the fermions have all disappeared.

This corresponds to the worldsheet action

$$
\frac{1}{4 \pi} \int d^{2} \sigma \sqrt{\hat{g}}\left\{\hat{g}^{a b} \eta_{\mu \nu} \partial_{a} X^{\mu} \partial_{b} X^{\nu}+2 X^{1} \hat{R}-\hat{\mu} X^{1} e^{2 X^{1}}+\kappa e^{2 X^{0}}\right\},
$$

where $\kappa$ is a constant that could be absorbed by a shift of $X^{0}$. Although this expression - and indeed the entire dictionary between free fermions and tachyons - was derived for weak tachyon fields, it is notable that (3.9) is in fact the direct sum of two noninteracting CFTs, and therefore provides an exact string tree-level solution.

The general solution (3.5) corresponds to a very narrow wedge of Fermi sea coming in from $-\infty$, opening up to the standard hyperbola at $t=T_{1}$ (we take $T_{1}<T_{2}$ for concreteness) and then closing back down at $t=T_{2}$. Solving for $p_{ \pm}$, and using equations (2.7) and (3.2), the tachyon field is

$$
T=-\hat{\mu} X^{1} e^{2 X^{1}}+\kappa\left(1-f\left(X^{0}\right)\right),
$$


where

$$
f(t)=\frac{\sinh \left(T_{2}-T_{1}\right)}{2 \cosh \left(t-T_{1}\right) \cosh \left(t-T_{2}\right)} .
$$

As before, the new term in the tachyon field has no spatial dependence. For $T_{1}<X^{0}<T_{2}$ it vanishes, and we recover regular Liouville theory (2.11). Let's take $T_{2}-T_{1} \gg 1$ so that the Liouville universe exists for an appreciable amount of time. Before and after this (arbitrarily long) period, the coupling becomes strong everywhere, and smooth spacetime disappears. This presumably corresponds to a process in which the tachyon is condensed in both the far future and the far past. Actually, equation (3.10) is meaningful only for small $X^{0}$ (for $T_{1}<X^{0}<T_{2}$ ), where the expression is

$$
T=-\hat{\mu} X^{1} e^{2 X^{1}}+\kappa\left(e^{2\left(X^{0}-T_{2}\right)}+e^{-2\left(X^{0}-T_{1}\right)}\right) .
$$

\subsection{Exact quantum solutions}

In this section we give exact quantum solutions with the prescribed semiclassical behavior. First we consider the general spacelike tachyon condensation of equation (3.5). We begin by introducing the dilation operator

$$
D_{\alpha}=e^{\frac{i \alpha}{2}(\hat{p} \hat{x}+\hat{x} \hat{p})}
$$

which stretches $x$ and shrinks $p$

$$
D_{\alpha} \hat{x} D_{-\alpha}=e^{\alpha} \hat{x}, \quad D_{\alpha} \hat{p} D_{-\alpha}=e^{-\alpha} \hat{p} .
$$

The hat on $\hat{x}$ and $\hat{p}$ indicates they are operators. Now consider the state at time $t=0$

$$
|\alpha, \mu\rangle=D_{\alpha}|\mu\rangle
$$

where $|\mu\rangle$ is the ground state (2.3), and the action $D_{\alpha}$ on a second quantized state is induced from its action on the first quantized wave functions, and can be written as

$$
D_{\alpha}=e^{-\frac{\alpha}{2} \int\left(d x \Psi^{\dagger}\left(x \partial_{x} \Psi\right)-\left(x \partial_{x} \Psi^{\dagger}\right) \Psi\right)}
$$

Since $D_{\alpha}$ does not commute with the Hamiltonian, $|\alpha, \mu\rangle$ is a time dependent state. At time $t=0$, in the semiclassical approximation, the Fermi surface is described by the equation

$$
e^{2 \alpha} p^{2}-e^{-2 \alpha} x^{2}=-\frac{1}{g_{s}}
$$


Taking $e^{-2 \alpha}=\tanh \frac{T_{2}-T_{1}}{2}$ and rescaling $g_{s}$ by $2 \sinh \frac{T_{2}-T_{1}}{2}$, we get the general solution (3.5) at time $t=\frac{1}{2}\left(T_{1}+T_{2}\right)$. Therefore, evolving $|\alpha, \mu\rangle$ with $H$ we get an exact quantum state whose Fermi surface evolves under time approximately according to (3.5). Fluctuations in this Fermi surface are suppressed, and the semiclassical limit approached, for example by taking $\mu$ to be very large.

The exact quantum state corresponding to (3.1) is even easier to find - it can be created by acting on $|\mu\rangle$ with the shift operator of the form $e^{-i \lambda_{-}(\hat{p}+\hat{x})-i \lambda_{+}(\hat{p}-\hat{x})}$.

Expansion around the static ground state of the matrix model has provided a powerful method of computing CFT correlators in the usual Liouville theory. Here we have presented simple exact quantum states related to the timelike bulk Liouville theory and the Sine-Gordon Liouville theory. Perhaps the corresponding matrix states will be of use in computing correlators of these CFTs.

\subsection{Stability}

The reader may be puzzled by the fact that the closed string tachyons in the Liouville theory are often referred to as massless, and thus can not condense in the usual sense. The possibility of tachyon condensation seems to be at odds with the stability of the $c=1$ matrix model. However the interactions $e^{X^{1} \pm X^{0}}$ and $e^{ \pm 2 X^{0}}$ are not normalizable fluctuations of the tachyon field. Therefore the state $|\mu\rangle$ does not decay via such fluctuations and there is no problem of stability.

\section{Closed cosmologies}

\subsection{Orbiting Fermi droplet}

In this subsection we construct a matrix cosmology from $N<\infty$ fermions in the $c=1$ matrix model. The construction will proceed in three steps.

In the first step we consider a state, involving an infinite number of fermions, in which every single-fermion eigenstate with energy greater than $-\mu$ is filled. Due to the Pauli exclusion principle, this state obeys

$$
b_{E}^{\dagger}|\tilde{\mu}\rangle=0, \quad E>-\mu, \quad b_{E}|\tilde{\mu}\rangle=0, \quad E<-\mu,
$$

where $b_{E}^{\dagger}$ creates a fermion of energy $E$. Redefining

$$
b_{E}^{\dagger} \rightarrow a_{E}, \quad b_{E} \rightarrow a_{E}^{\dagger}
$$


we find that $|\tilde{\mu}\rangle$ obeys

$$
a_{E}|\tilde{\mu}\rangle=0, \quad E>-\mu, \quad a_{E}^{\dagger}|\tilde{\mu}\rangle=0, \quad E<-\mu,
$$

which is just the usual condition for a state whose Fermi sea is filled up to energy $\mu$. Hence the state (2.3) is equivalent to the usual matrix model ground state (up to a shift and sign reversal of the energy implied by (4.2)). Excitations of this state can be described by a propagating tachyon. This is just the usual particle-hole duality.

Next we consider the state

$$
\begin{aligned}
& b_{E}^{\dagger}\left|\mu_{1}, \mu_{2}\right\rangle=0, \quad-\mu_{2}<E<-\mu_{1}, \\
& b_{E}\left|\mu_{1}, \mu_{2}\right\rangle=0, \quad E<-\mu_{2}, \quad E>-\mu_{1}, \quad \mu_{1}<\mu_{2} .
\end{aligned}
$$

This state has two Fermi surfaces, one at $\mu_{1}$ and the other at $\mu_{2}$. Small perturbations of the Fermi surfaces are classically decoupled. This means that the tree level tachyon S-matrix of this system, which in turn determines the full perturbative S-matrix via unitarity, is equivalent to two decoupled $c=1$ matrix models, one with coupling $g_{1}=1 / 2 \mu_{1}$ the other with coupling $g_{2}=1 / 2 \mu_{2}$. Outside of perturbation theory, large excitations with energies of (absolute value) of order $1 / g_{i}$ can couple the two parallel universes. This is similar to the statement that large energies in the usual matrix model can splash over the potential barrier.

In the last step we make a single universe with a finite number of fermions and a finite spatial volume. A semiclassical description of this state, which we denote $\mid$ crescent $\rangle$, is obtained at $t=0$ by simply terminating and connecting the upper and lower Fermi surfaces at some (large) value of the spatial coordinate $x=x_{\max }$. The filled crescentshaped region of phase space at $t=0$ is depicted in figure $4(\mathrm{a})$. The finite number of fermions is given by the finite volume of phase space. A picture of the filled energy region is given in figure 4(b). |crescent $\rangle$ is time dependent. This region of phase space follows along classical trajectories, sliding around the hyperbolae and then heading out to $x=\infty$ in an elongated shape.

What is the physical interpretation of the state $\mid$ crescent $\rangle$ ? It can be found by taking $x_{\max }$ to be extremely large so that it is far out in the weakly coupled asymptotic region. Then we can consider scattering experiments in which we scatter tachyons off the Liouville wall from some fixed spatial position $x_{s} \ll x_{\max }$ but still deep in the weak coupling region. Clearly such experiments will not be affected by the termination of the Fermi sea at $x_{\max }$. 


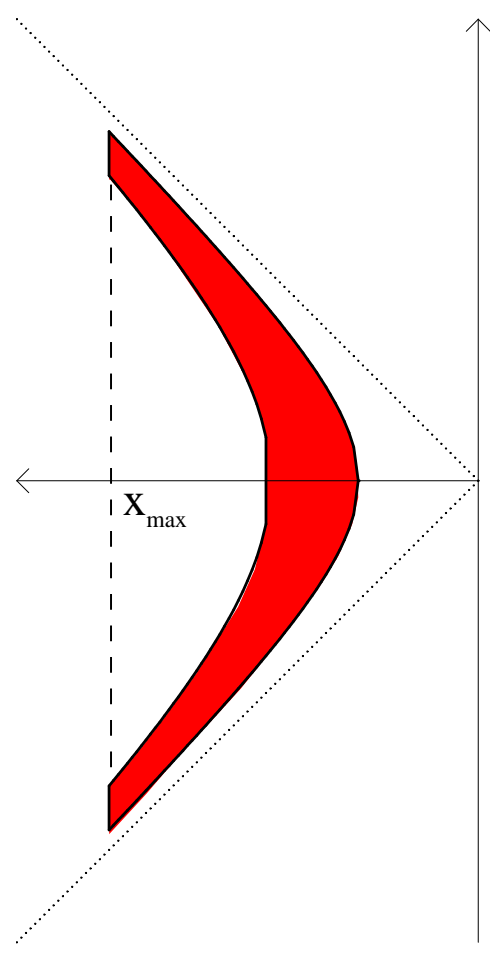

(a)

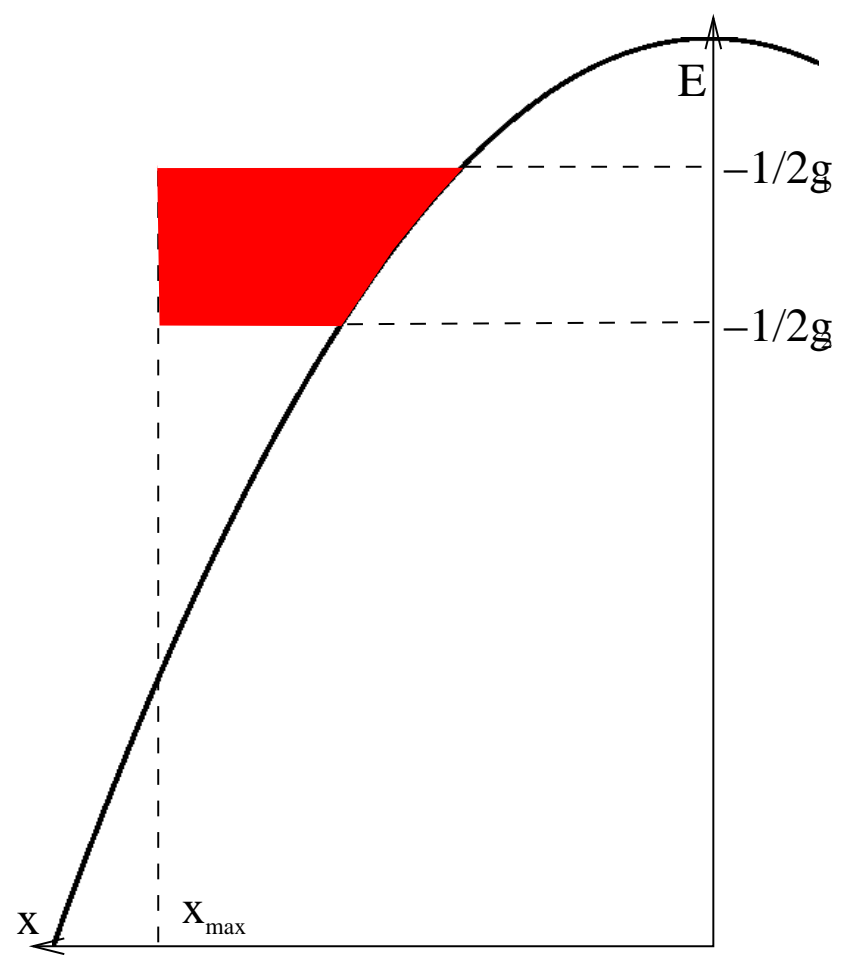

(b)

Fig. 4: (a) Phase space diagram of the Fermi sea at $t=0$ for the crescent cosmology. (b) In a position-energy diagram at $t=0$ the potential is filled up to $x=x_{\max }$.

Therefore at, $t=0$, we can to a good approximation describe the system as two universes, each of which has a tachyon wall at one end and is joined to the other at the large position $x_{\max }$. The stringy junction at which the two universes meet involves energies of order $\frac{1}{g}$ and has no perturbative description that we know of.

As $t$ evolves forward in time, the termination points of the Fermi sea do not remain at the same spatial position. The lower termination point - which we refer to as the head of the universe - moves away from the Liouville wall at the speed of light. The "tail" of the universe initially moves toward the Liouville wall, but then bounces off of it towards spatial infinity. In the far future both the head and the tail of the universe - i.e. the junctions between the parallel universes - are headed away from the Liouville wall at the speed of light. The only tachyon excitations which survive are those which are also moving at the speed of light sandwiched between the head and tail. The Penrose diagram of this cosmology is depicted in figure 5 .

At late and early times, the universe (with the exception of the defect region) is at weak coupling. If the tachyon wall is at strong coupling, it bounces through a strong 


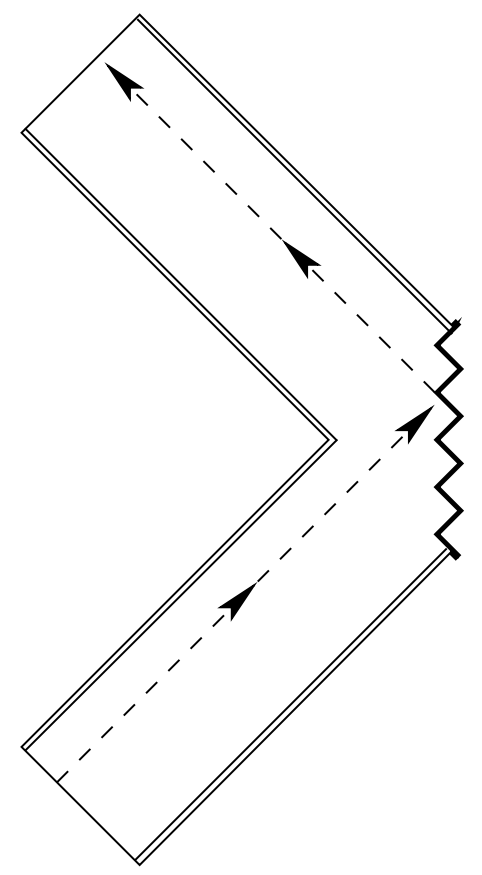

Fig. 5: The Penrose diagram for the crescent cosmology consists of two null strips joined at the Liouville wall. Each point on the diagram corresponds to two points (one in each universe), except for the double-lined edges, at which the two universes join. Tachyon excitations can scatter from past to future null infinity.

coupling phase to get from one weakly coupled region to the other. In the tachyon picture this will look like a universe which bounces through a singularity.

An analog of the bosonization formula (2.7) can be worked out here and leads to two chiral, rather than one nonchiral, fields. Consider part of a Fermi sea sandwiched between two hyperbola defined by $x^{2}-\left(p_{i}\right)^{2}=g_{i}^{-1}$ where $i=1,2$ and $g_{1}>g_{2}$. We will be interested in the $p_{i}>0$ and large $-x$ region, corresponding to the far past of the $\mid$ crescent $\rangle$ state (the treatement of the far future is analogous). Allowing $p_{i}$ to fluctuate around this solution, the equation of motion is

$$
\partial_{t} p_{i}=x-\left(\partial_{x} p_{i}\right) p_{i}
$$

and the commutation relationship is

$$
\left[p_{i}(x), p_{j}\left(x^{\prime}\right)\right]=2 \pi i(-)^{j} \delta_{i j} \partial_{x} \delta\left(x-x^{\prime}\right)
$$

Define two new fields, $A_{i}(q)$, where $x=-e^{-q}$, by

$$
p_{i}=e^{-q}-e^{q} A_{i} .
$$


The equation of motion for these two fields is

$$
\left(\partial_{q}-\partial_{t}\right) A_{i}=0
$$

so they are chiral (right-moving) fields. They are linked by a commutation relationship

$$
\left[A_{i}(q), A_{j}\left(q^{\prime}\right)\right]=2 \pi i \delta_{i j}(-)^{j} \partial_{q} \delta\left(q-q^{\prime}\right)
$$

These are the commutation relation for the (derivatives of) two chiral bosons, one with positive and one with negative norm. Thus, the bosonic interpretation of this early-time patch of Fermi sea is that it corresponds to two chiral, right-moving fields, consistent with the analysis above.

\subsection{Validity of the semiclassical approximation}

The previous section described quantum states of free fermions as regions of Fermi liquid moving under Hamiltonian flow in phase space. Of course there are many families of exact quantum states with any prescribed semiclassical limit. For our purposes so far it has been important to know that there do exist exact quantum states whose semiclassical limit has the requisite properties, but it was unnecessary to specify which one we were considering.

However we would like to know when the semiclassical description is valid and how or when it breaks down. Validity of the semiclassical approximation requires that the size of the filled region of phase space in units of $\hbar$ is large (in the preceding sections $\hbar$ has been set to one). Equivalently the number of fermions must obey $N \gg 1$. In addition the Fermi surface must vary slowly on the scale set by $\hbar$. Another way of saying this is that quantum effects produce a coarse graining of size $\hbar$ in all the phase space diagrams.

For the ground states $|\mu\rangle$, this is equivalent to the perhaps more familiar statement that $g_{s} \sim 1 / \mu \ll 1$. The classical hamiltonian flow in phase space is invariant under a scaling symmetry $x \rightarrow e^{\rho} x, \quad p \rightarrow e^{\rho} p$ and $E \rightarrow e^{2 \rho} E$. Therefore scaling $g_{s} \sim 1 / E$ is the same as scaling the size of fundamental phase space cells, and $g_{s} \rightarrow 0$ is the semiclassical Fermi liquid limit.

More generally, given any trajectory of a droplet of Fermi liquid in phase space with surface $p(x)=f(x, t)$, there is a family of droplets $p(x)=e^{-\rho} f\left(e^{\rho} x, t\right)$ containing of order $e^{2 \rho}$ fermions. The semiclassical expansion is an expansion in $e^{-\rho}$. It can also be thought of as an expansion in the size of the phase space cells. 
The validity of the semiclassical approximation at any moment in time does not guarantee its eternal validity. Consider for example the crescent cosmology of figure 4(a). In the far future, the filled region of phase space stretches in the horizontal $(x)$ direction, while squeezing in the vertical $(p)$ direction. Depending on the precise form of the initial quantum state, the uncertainly in $p$ could come to dominate over the semiclassical spread of $p$, and the state would cease to behave semiclassically. While we have not studied this question in detail, clearly the very long time behavior of the cosmologies discussed herein could differ from the semiclassical picture.

\section{General droplet cosmology}

The construction of the previous section has many generalizations. In order to better understand what kind of universes can be constructed from the Fermi sea filling various regions in phase space, in this section we discuss a few aspects of the Hamiltonian flow in the $(x, p)$ plane implied by the Hamiltonian $H=\frac{1}{2}\left(p^{2}-x^{2}\right)$.

The Fermi surface moves following the equations of motion $\dot{p}=x$ and $\dot{x}=p$. Consider a pair of points $\left(x_{1}, p_{1}\right)$ and $\left(x_{2}, p_{2}\right)$. The slope of the line joining them

$$
\alpha \equiv\left(p_{1}-p_{2}\right) /\left(x_{1}-x_{2}\right)
$$

evolves according to the equation

$$
\dot{\alpha}=1-\alpha^{2} .
$$

The slope evolves independently of its absolute position. As a result, straight lines stay straight and parallel ones stay parallel. The solution is either

$$
\alpha(t)=\operatorname{coth}\left(t-t_{0}\right)
$$

for $|\alpha|>1$ or

$$
\alpha(t)=\tanh \left(t-t_{0}\right)
$$

for $|\alpha|<1$. Take a curve in the $x=p$ plane at some instant, and consider its evolution. We will divide the curve into two regions: Steep and Shallow. The Steep part has $\mid$ slope $\mid>1$ and the Shallow $\mid$ slope $\mid<1$. At $t=-\infty$, the entire Steep region has slope arbitrarily close to -1 . At the curve evolves from $t=-\infty$ to $t=+\infty$, the point at which it is vertical moves through the Steep region, and eventually, at $t=+\infty$, the entire region has slope arbitrarily close to 1 . This is the type of region we have focused on up to this point. 
The (almost) straight shape at $t= \pm \infty$ corresponds to the flat weak coupling region in the Liouville model. Every small perturbation about these asymptotic shapes must pass through the vertical point on the Fermi surface, where the collective fields are strongly coupled. This corresponds to scattering off the Liouville wall. By a small perturbation we mean here one in which the absolute value of the slope remains greater than one, i.e. a perturbation that does not create a Shallow region within the Steep region.

The Shallow region is quite different: it's slope remains finite. It is not clear what the space-time interpretation of the collective coordinates describing the fluctuations about this region is. Classically, the Steep and the Shallow regions evolve independently, and fluctuations do not move from one to the other. Quantum mechanically, these region do interact, corresponding to stringy interaction in the bosonic picture.

Consider in this light the state $\mid$ crescent $\rangle$ described in the previous section. It contains two Steep regions, and the physical interpretation of these was given above. These two steep regions must be connected by Shallow regions. The mysterious stringy connection between the two parallel universes happens at the Shallow regions.

One way to avoid having a Shallow region in the $\mid$ crescent $\rangle$ picture is to join the two Steep regions with a cusp. The two parallel universes are then joined through some sort of stringy defect at the cusp.

The Shallow region need not be small compared to the Steep region. We expect that the evolution of a fairly general Fermi sea droplet corresponds to a closed cosmology. Such a droplet can be divided into four pieces: the Right and Left Steep regions, and the Top and Bottom Shallow regions, as illustrated in figure 6.

The physics in the asymptotic past/future is similar to that of the |crescent $\rangle$ shape, though with all four regions contributing to large portions of the universe. In the asymptotic future, for example, the Right and the Bottom regions form a single region, which should be described as a whole by the standard methods used so far.

It would be interesting to discuss such a closed cosmological universe in the same detail as we have been able to discuss the universe with the moving Liouville wall arising from the draining of the Fermi sea. As a step in this direction, we give a simple solution of the flow equations which corresponds to such a droplet

$$
e^{-2 t}\left(x+p+e^{t}\right)^{2}+e^{2 t}\left(x-p+e^{-t}\right)^{2}=a^{2} .
$$

This is only one example from a large family of similar solutions. For small enough $a$ it describes a moving and evolving ellipse which at time $t=0$ is a circle of centered at 


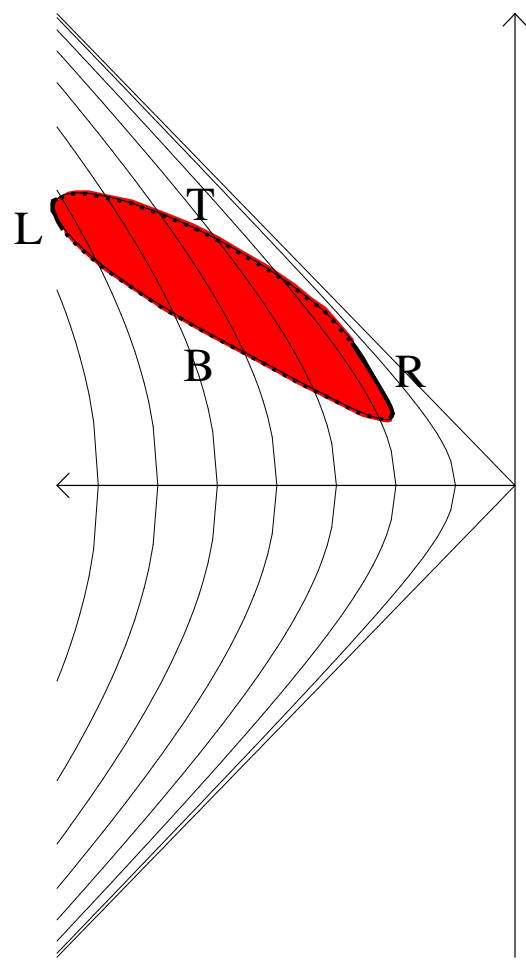

(a) Before

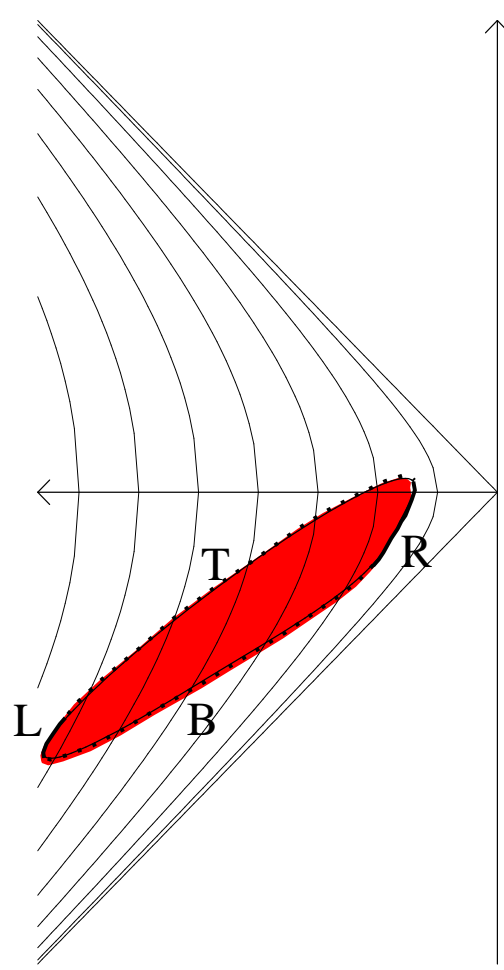

(b) After

Fig. 6: A fairly general Fermi droplet can be divided into four regions that behave differently under evolution.

$(x, p)=(-1,0)$. It would be quite interesting to obtain the tachyon field corresponding to this solution, but the technique used in this paper does not seem to apply.

As a last comment, one might also consider solutions which extend over the top of the Fermi sea. Trajectories in the upper quadrant of phase space are related by a canonical transformation $x \leftrightarrow p$ to those in the left quadrant, so these should also have a semiclassical interpretation in some regions.

\section{SD-branes and higher dimensions}

The cosmologies of the preceding section can be viewed as a "near-horizon" decoupling limit of a stack of $N c=1 \mathrm{sD}$-branes. Indeed this project arose in an attempt to understand such a decoupling limit for sD-branes. Viewed in this way, our results may have a generalization to higher dimensions. In this section we explain this connection.

In critical string theory the spectrum of open strings on an unstable D-brane has a tachyon field $T_{\text {open }}$ which lives on the boundary of the string worldsheet. This has sbrane solutions [27] which are represented [28] by the boundary CFT interaction $T_{\text {open }} \sim$ 
$\lambda \cosh X^{0}$. When $\lambda=\frac{1}{2}$, this interaction rotates Neumann to Dirichlet boundary conditions and the s-brane becomes an array of sD-branes located on the imaginary time axis. The sD-brane array state is perturbatively dual to a state $|C\rangle$ which is a particular configuration of imploding and exploding closed strings [29,30].

A first question to ask is: what is the $c=1$ analog, in the free Fermi picture, of the closed string state $|C\rangle$ ? According to the picture developed in [8 10], in the semiclassical limit an unstable D0-brane with $T_{\text {open }} \sim \lambda \cosh X^{0}$, is represented in the free fermion picture by a single fermion orbiting above the Fermi sea. When $\lambda=\frac{1}{2}$, the fermion energy is such that it skims the surface of the Fermi sea and can be described as a closed string excitation. Hence the $c=1$ analog of the sD-brane array state is a single fermion skimming the surface of the Fermi sea.

In any dimension, it is of interest to try to find a large $N$ decoupling limit of a stack of $N$ sD-branes, which may be holographically dual to a supergravity solution. For $c=1$, taking $N$ sD-branes corresponds to taking $N$ fermions clustered together. This indeed has a nice semiclassical limit which we described above as an isolated droplet of Fermi liquid. For a droplet orbiting above a bulk Fermi sea, it is a decoupling limit because small fluctuations of the bulk and droplet components of the Fermi surface do not talk to one another. The construction of such a decoupling limit in the higher dimensional case is an intriguing open challenge.

\section{Acknowledgements}

This work was supported in part by DOE grant DE-FG02-91ER40654 and the Harvard Society of Fellows. We are grateful to P.-M. Ho, V. Kazakov, J. Maldacena, J. McGreevy, S.Minwalla, J. Polchinski, N. Seiberg, S. Shenker and T. Takayanagi for useful conversations. 


\section{References}

[1] M. R. Douglas and S. H. Shenker, "Strings In Less Than One-Dimension," Nucl. Phys. B 335, 635 (1990).

[2] D. J. Gross and A. A. Migdal, "Nonperturbative Two-Dimensional Quantum Gravity," Phys. Rev. Lett. 64, 127 (1990).

[3] E. Brezin and V. A. Kazakov, "Exactly Solvable Field Theories Of Closed Strings," Phys. Lett. B 236, 144 (1990).

[4] D. J. Gross and N. Miljkovic, "A Nonperturbative Solution of $D=1$ String Theory," Phys. Lett. B 238, 217 (1990);

[5] E. Brezin, V. A. Kazakov and A. B. Zamolodchikov, "Scaling Violation in a Field Theory of Closed Strings in One Physical Dimension," Nucl. Phys. B 338, 673 (1990);

[6] P. Ginsparg and J. Zinn-Justin, "2-D Gravity + 1-D Matter," Phys. Lett. B 240, 333 (1990).

[7] G. W. Moore, M. R. Plesser and S. Ramgoolam, "Exact S matrix for 2-D string theory," Nucl. Phys. B 377, 143 (1992) arXiv:hep-th/9111035].

[8] J. McGreevy and H. Verlinde, "Strings from tachyons: The c $=1$ matrix reloaded," arXiv:hep-th/0304224.

[9] E. J. Martinec, "The annular report on non-critical string theory," arXiv:hepth/0305148.

[10] I. R. Klebanov, J. Maldacena and N. Seiberg, "D-brane decay in two-dimensional string theory," JHEP 0307, 045 (2003) arXiv:hep-th/0305159.

[11] T. Takayanagi and N. Toumbas, "A matrix model dual of type 0B string theory in two dimensions," JHEP 0307, 064 (2003) arXiv:hep-th/0307083.

[12] M. R. Douglas, I. R. Klebanov, D. Kutasov, J. Maldacena, E. Martinec and N. Seiberg, "A new hat for the $c=1$ matrix model," arXiv:hep-th/0307195.

[13] J. Polchinski, "Classical Limit Of (1+1)-Dimensional String Theory," Nucl. Phys. B 362, 125 (1991).

[14] S. R. Das and A. Jevicki, "String Field Theory And Physical Interpretation Of D = 1 Strings," Mod. Phys. Lett. A 5, 1639 (1990).

[15] D. Minic, J. Polchinski and Z. Yang, "Translation Invariant Backgrounds In (1+1)Dimensional String Theory," Nucl. Phys. B 369, 324 (1992).

[16] S. Y. Alexandrov, V. A. Kazakov and I. K. Kostov, "Time-dependent backgrounds of 2D string theory," Nucl. Phys. B 640, 119 (2002) arXiv:hep-th/0205079.

[17] S. Alexandrov and V. Kazakov, "Correlators in 2D string theory with vortex condensation," Nucl. Phys. B 610, 77 (2001) arXiv:hep-th/0104094.

[18] S. Y. Alexandrov and V. A. Kazakov, "Thermodynamics of 2D string theory," JHEP 0301, 078 (2003) arXiv:hep-th/0210251. 
[19] S. Y. Alexandrov, V. A. Kazakov and I. K. Kostov, "2D string theory as normal matrix model," Nucl. Phys. B 667, 90 (2003) arXiv:hep-th/0302106.

[20] J. Polchinski, "What is string theory?," arXiv:hep-th/9411028.

[21] M. Natsuume and J. Polchinski, "Gravitational Scattering In The $\mathrm{C}=1$ Matrix Model," Nucl. Phys. B 424, 137 (1994) arXiv:hep-th/9402156].

[22] I. R. Klebanov, "String theory in two-dimensions," arXiv:hep-th/9108019.

[23] P. Ginsparg and G. W. Moore, "Lectures On 2-D Gravity And 2-D String Theory," arXiv:hep-th/9304011.

[24] B. C. Da Cunha and E. J. Martinec, "Closed string tachyon condensation and worldsheet inflation," arXiv:hep-th/0303087.

[25] A. Strominger and T. Takayanagi, "Correlators in timelike bulk Liouville theory," arXiv:hep-th/0303221.

[26] V. Schomerus, "Rolling tachyons from Liouville theory," arXiv:hep-th/0306026.

[27] M. Gutperle and A. Strominger, "Spacelike branes," JHEP 0204, 018 (2002) arXiv:hep-th/0202210.

[28] A. Sen, "Rolling tachyon," JHEP 0204, 048 (2002) arXiv:hep-th/0203211.

[29] A. Maloney, A. Strominger and X. Yin, "S-brane thermodynamics," arXiv:hepth/0302146.

[30] D. Gaiotto, N. Itzhaki and L. Rastelli, "Closed strings as imaginary D-branes," arXiv:hep-th/0304192. 